\title{
HACIA UNA NUEVA GOBERNANZA: LOS PODERES PÚBLICOS Y EL TERCER SECTOR. UNA RELACIÓN NECESARIA PERO INEXISTENTE
}

\author{
TOWARDS A NEW GOVERNANCE: PUBLIC AUTHORITIES AND THIRD \\ SECTOR. A NECESSARY BUT NON-EXISTENT RELATIONSHIP
}

\author{
Verónica Gómez Calvo \\ Adela Romero Tarín \\ Universidad de Alicante. España/Spain \\ veronica.gomez@usac.unr.edu \\ adela.romero@ua.es
}

Recibido/Received: 27/12/2015

Modificado/Modified: 13/05/2016

Aceptado/Accepted: 20/09/2016

\section{RESUMEN}

Estos últimos años, la sociedad capitalista ha entrado en una profunda recesión. El Estado y los ciudadanos se encuentran cada vez más alejados entre sí, y los partidos tradicionales no cumplen su función de representatividad de las necesidades de los ciudadanos. Europa se encuentra inmersa en una crisis económica, pero también en una crisis de valores. Las balanzas entre el desarrollo económico y el social no están equilibradas (Massé, 1964), y la reinvención de un nuevo modelo de Estado, es ya es una necesidad. Fruto de los procesos de globalización, el papel del Estado y la participación ciudadana se han ido marginando y subordinando cada vez más a los dictámenes del mercado. Este artículo tiene el propósito de analizar un nuevo concepto de Gobernanza, en base a las relaciones entre Estado, mercado, Tercer Sector, y ciudadanía con el objetivo de esclarecer cuál es la mejor manera de dar respuesta a las necesidades sociales. Además en él, se hace hincapié en los conceptos de diálogo y participación ya que estos muestran la relación entre el Estado, el mercado y la ciudadanía, a través de una eficaz y eficiente orientación e intervención del Estado en su forma de gobernar (Canales, 2002).

\section{PALABRAS CLAVE}

Tercer Sector, economía social, Gobernanza, participación ciudadana, diálogo social, diálogo civil, diálogo institucional, mercado.

\section{SUMARIO}

1. Introducción. 2. Los objetivos de la investigación. 3. El mercado y su papel en la descohesión y en la fractura social. 4. La Sociedad Civil se organiza para dar respuesta a los problemas sociales: El Tercer Sector. 5. El diálogo social y la participación ciudadana como elementos del cambio. 6. El diálogo civil y el partenariado en la actualidad. 7. Los resultados y las principales recomendaciones. 8. Conclusiones. 9. Bibliografía.

\section{ABSTRACT}

In recent years, capitalist society has entered a deep recession. The state and its citizens are increasingly far apart, and the traditional parties do not fulfill their function of representation of the needs of citizens. 
Europe is undergoing an economic crisis but also a crisis of values. The balance between economic and social development are not balanced (Massé, 1964), and the reinvention of a new model of state, is already a necessity. The result of the processes of globalization, the role of government and citizen participation have been marginalized and increasingly subordinated to the dictates of the market. This paper aims to analyze a new concept of governance based on the relationship between state, market, Third Sector, and citizenship in order to clarify what is the best way of responding to social needs. The emphasis on the concepts of dialogue and participation as these show the relationship between the state, the market and the public, through an effective and efficient guidance and intervention in its governance (Canales, 2002).

\section{KEYWORDS}

Third Sector, social economy, Governance, citizen participation, social dialogue, civil dialogue, dialogue institutional, market.

\section{CONTENTS}

1. Introduction. 2. Research objectives. 3. The market and its role in social decohesion and fracture.

4. Civil society is organized to respond to social problems: The Third Sector. 5. Social dialogue and citizen participation as elements of change. 6. Civil dialogue and partnership at present. 7. Results and conclusions. 8. Conclusions. References.

\section{INTRODUCCIÓN}

El modelo económico actual, en su forma capitalista más pura y con pocos contrapesos sociales, muestra sus caras más amargas, y entre otras destacaría: la extensión del desempleo, la pobreza y la exclusión social, la destrucción del entorno el medioambiental y el cambio climático, etc. De esta situación se deriva el nacimiento del Tercer Sector, que surge a raíz de ofrecer éste, respuestas a los problemas que no son solventados ni por las insuficientes políticas públicas, ni por el criterio de dejar hacer al mercado. Sus valores y objetivos sociales son por ello un "aliado natural" de los Poderes Públicos, especialmente, a nivel territorial, local.

Además, el sistema capitalista neoliberal ha ido restringiendo cada vez más el papel del Estado. Si hasta bien entrada la década de los 70 había un contrapeso político frente a las corporaciones a través del Estado, los sindicatos y los programas sociales, no se corrió la misma suerte a partir de los años 80, cuando el neoliberalismo empezó a imponerse como política económica. Desde el momento en que, con ayuda de los avances tecnológicos en las telecomunicaciones y los transportes y la liberalización de los capitales, están en disposición de invertir su dinero allí dónde lo consideren preciso, instalando o desinstalando empresas, generando o destruyendo empleo y afectando en su conjunto a la balanza de pagos de un país, las multinacionales se imponen a los gobiernos en numerosas materias como la inversión, la localización industrial, el empleo, los tipos de interés, fiscalidad, y un largo etcétera (Alós y Jodar, 1996: 22). Lo anterior señalado, ha significado un claro repliegue del Estado y, sobre todo, de la fuerza de trabajo frente al capital.

Este repliegue ha producido un claro distanciamiento entre los Poderes Públicos y la sociedad, que apenas participa en la vida pública y las votaciones como consecuencia del descrédito de la política y sus instituciones públicas. Por todo ello, es necesario un cambio de paradigma del diálogo entre los Poderes Públicos y el Tercer Sector, y habilitar plataformas y nuevas vías para incluir en la planificación de políticas públicas efectivas y exitosas a la sociedad civil. Aquí no hablamos de mero diálogo social en forma de consultas, pues se 
requieren políticas públicas que integren planteamientos y demandas desde la sociedad civil si pretendemos resolver problemas coyunturales en lugar de poner meros parches puntuales.

\section{LOS OBJETIVOS DE LA INVESTIGACIÓN}

Este artículo pretende analizar tres cuestiones:

1. Las relaciones entre Estado, mercado, Tercer sector y sociedad civil, y cómo éstas desembocan en soluciones o problemas sociales.

2. Un nuevo concepto de gobernanza que aporte políticas públicas y soluciones eficaces a los problemas sociales.

3. Una serie de recomendaciones para que esta gobernanza sea una realidad.

\section{EL MERCADO Y SU PAPEL EN LA DESCOHESIÓN Y EN LA FRACTURA SOCIAL}

Karl Polanyi ya hizo notar que ninguna sociedad anterior a la sociedad industrial del siglo XIX había adoptado "la ganancia" como único criterio de justificación para la actividad económica y el comportamiento en la vida cotidiana. El concepto de "mercado autorregulado" se deriva de este criterio, con lo que en la actualidad vamos en el camino de cumplir la profecía de Karl Polanyi: "permitir a los mecanismos del mercado dirigir el destino del mundo desembocará en la demolición de la sociedad" (George, 1999: 1).

La sociedad industrial creó unos mecanismos de exclusión social, que han ido adquiriendo más fuerza según se desarrollaban. Por ejemplo, se diseñaron los "perfiles profesionales" que surgieron de tres conceptos básicos: a) Lo importante es ser eficaz y rentable para el sistema; b) Las personas que trabajan sólo han de limitarse a lo que se les pide; y c) Las personas que no cumplen con estas normas son excluidas del proceso de selección, y por tanto, excluidos del mercado laboral. Es decir, personas enfermas, discapacitadas, ocupadas en el cuidado de dependientes, etc. son excluidas del modelo productivo donde son vistos como mera mercancía productiva. Era el comienzo de la exclusión laboral, pero con ella nace la exclusión social ya que en la sociedad industrial todo se piensa desde lo productivo. Aunque se han superado muchos aspectos de ésta, su fuerza de exclusión sigue vigente ya que aúna un perfil profesional definido desde criterios poco integradores que generan exclusión laboral (y social) con una estrategia de acumulación de capital que conlleva a maximizar beneficios a costa de la fractura social. El problema del trabajo es que se gestiona desde lo productivo, sometiendo el destino de las personas a las leyes del mercado.

Por otro lado, el "mercado" es un conjunto de disposiciones que incluye leyes, convenciones, la infraestructura física y social, y la distribución de riqueza y la propiedad, sobre cuya base se realizan los intercambios. Otro efecto importante que opera en el mercado, es el poder de compra. Cuando hay mucha oferta y es equitativa, el mercado no funciona igual que cuando no hay mucha oferta y la capacidad de compra es muy desigual, por lo que el mercado tiene mucha fuerza para determinar los sueldos y la distribución de la riqueza de un país. No obstante, la teoría neoliberal presenta a los mercados como "un fenómeno natural", cuando no es sino consecuencia del ejercicio de poder del Estado u otras instancias supraestatales que instituyen estos mercados. Ni son "neutrales", pues las reglas del juego que dictan cómo deben funcionar, se deciden antes de que los mercados empiecen a operar, beneficiando a ciertos colectivos frente a otros. 
El mercado refleja en realidad, una estructura determinada de relaciones de poder, en las que las agencias estatales asumen la función de hacer cumplir acuerdos y de ejercer como árbitro de disputas, siendo garantes del adecuado funcionamiento de unos mercados que han sido instituidos previamente en relación a los intereses de ciertos grupos con poder de presión (MacWean, 2001:178). De hecho, el mercado por sí sólo no puede solventar problemas como la pobreza, las desigualdades, la precariedad laboral, la carencia de servicios sanitarios o infraestructuras, etc. porque son problemas que tienen su raíz en unas relaciones de poder que están institucionalizadas y, esto sólo se puede resolver incrementando la participación democrática ciudadana, de manera que se re-equilibren las relaciones de poder.

Además, en la actualidad, podemos hablar ya no de meros "mercados", sino de sistemas de mercados, convirtiéndose en el factor principal de todos los asuntos económicos. Pero cuando una sociedad se desarrolla en un sistema de sistemas de mercados, se gestiona como un complemento del sistema de mercado mismo, supeditando el conjunto de la sociedad, incluida la política, a su buen funcionamiento (Polanyi, 1944:57).

\section{LA SOCIEDAD CIVIL SE ORGANIZA PARA DAR RESPUESTA A LOS PROBLEMAS SOCIALES: EL TERCER SECTOR}

Precisamente, como respuesta a los problemas sociales, surgieron los primeros sindicatos del siglo XIX y los movimientos sociales, así como la Economía Social, todos ellos con la clara intención de neutralizar los efectos no deseados y el abuso de poder en las relaciones laborales de la Revolución industrial. La Economía Social del siglo XIX aglutinaba, principalmente, a cooperativas, mutualidades y asociaciones, el núcleo duro de la misma. En los años 70, volvió a resurgir de nuevo con fuerza debido a la crisis del Estado de Bienestar, la falta de empleo, y el riesgo de exclusión del mercado de trabajo.

Esta situación antedicha, originó además formas dinámicas de autoempleo y reforzó la solidaridad entre los actores económicos para hacer frente a las nuevas necesidades o aquellas que no podían ser satisfechas, principalmente, las de las poblaciones más desfavorecidas. Desde entonces, han ido apareciendo múltiples tipos de organizaciones que adoptan formas jurídicas y organizaciones múltiples, a veces distintas de las formas clásicas de la Economía Social.

Estas empresas y organizaciones desarrollan su actividad entre la economía pública y la economía capitalista tradicional, combinando múltiples recursos mercantiles y no mercantiles que van desde donaciones filantrópicas, subvenciones del Estado, e ingresos mercantiles, hasta el trabajo voluntario y el remunerado. De esta manera, integran la lógica del mercado junto a la solidaridad y la redistribución, adoptando formas internas de gestión propias de las empresas mercantiles o a las asociaciones cívicas tradicionales del Tercer Sector.

Las cooperativas, mutuales y asociaciones, son las organizaciones clásicas de la Economía Social en los países del Norte, "su ética se traduce por los principios siguientes: la finalidad de servicio a los miembros o la colectividad en lugar del beneficio, la autonomía de gestión, el proceso de decisión democrática, y la preeminencia de las personas y del trabajo sobre el capital en la distribución de los ingresos" (Defourny, Develtere, Fonteneau, 1999:38). Pero además, existen otras organizaciones de mercado y de no-mercado que completan el Tercer Sector con el mismo objetivo de construir cohesión social: empresas sociales, ONG's, empresas de la economía solidaria, banca ética, fondos solidarios, etc.

En todas ellas, el objetivo no está en las ganancias obtenidas por el producto o servicio, aunque sean entidades de mercado en algunos casos, y sea necesaria la viabilidad y 
sostenibilidad económica en todas ellas, sino aportar soluciones para colectivos de personas excluidas o cubrir demandas que de otra manera no sería posible cubrir. Es decir, asumen asuntos y actividades de interés general que deberían ser asumidos por el propio Estado. El sector privado no suele percibir estas necesidades y no es siquiera, en muchas ocasiones, consciente de que pueda provocar exclusión social y pobreza, por lo que a los excluidos sociales sólo les queda ser atendidos por el Tercer Sector.

El objetivo principal del Tercer Sector los ubica junto a las entidades definidas como "no lucrativas", en una situación funcional y material similar a las Administraciones, precisamente por realizar "servicios de interés general" a la sociedad.

La base para esta identidad es que cumplan las condiciones de:

a) Servir de complemento y coadyuvar a la prestación de actividades de interés general que, tradicionalmente, deberían ser realizadas constitucionalmente por los Entes Públicos (salud, deporte, medioambiente, vivienda, tercera edad, juventud, infancia, discapacitados sensoriales y psíquicos, etc.) y

b) Suplir insuficiencias de los Poderes Públicos o gestionar de forma ágil nuevas demandas sociales (Castro Sanz, 2014:28).

Según el Informe de CIRIEC, presentado al Consejo Económico y Social Europeo (CESE), la economía social -y se puede extrapolar al Tercer Sector perfectamente-, ha demostrado la capacidad de incrementar el nivel de cohesión social a través de la integración laboral de personas y territorios desfavorecidos, reduciendo los niveles de pobreza y exclusión. Así mismo, incrementa la cultura democrática y dinamiza la participación social porque da voz y capacidad de negociación a grupos antes excluidos del proceso económico y del proceso de elaboración y aplicación de políticas públicas, particularmente, a nivel regional y local.

En realidad, si queremos políticas públicas eficaces, deben ser consensuadas y construidas entre el Estado y la sociedad civil organizada bajo el paraguas del Tercer Sector. Pero en su lugar, lo que se evidencia es la estrechez de la democracia participativa y escasez de estructuras que habiliten este diálogo que además no puede reducirse a meras consultas, sino a diálogos sistemáticos a lo largo del proceso de planificación, ejecución, seguimiento y evaluación de las decisiones adoptadas.

El diálogo social está reducido en la actualidad a los Poderes Públicos, los empresarios y los trabajadores, pero hoy en día, la economía y la sociedad son más plurales y exigen implicar a una gran variedad de actores socio-económicos, ya que contribuyen significativamente a la vertebración del territorio, del tejido asociativo y al desarrollo de la democracia participativa. Excluir a estos protagonistas, sería excluir precisamente a los actores que convergen en soluciones de justicia social.

El mismo Parlamento Europeo apela a los representantes de la sociedad europea a participar en el diálogo civil, así como en la elaboración de políticas y programas europeos, permitiendo influir en la toma de decisiones. Sin embargo, hasta la fecha, no hay mecanismos que aseguren este diálogo ni se están construyendo plataformas sistemáticas que lo normalicen y lo sistematicen, aunque se presenta como la única manera de formular propuestas a largo plazo que resuelvan problemas sociales, de recuperar la credibilidad institucional y construir una democracia participativa inexistente en la actualidad. 


\section{EL DIÁlOGO SOCIAL Y LA PARTICIPACIÓN CIUDADANA COMO ELEMENTOS DEL CAMBIO}

A partir de la Revolución Francesa, los conceptos de libertad, fraternidad e igualdad, surgieron y se difundieron con gran intensidad, reclamando su regulación, presencia y defensa. Esta situación de lucha y reivindicación popular, continuó durante el siglo XVIII y XIX, y transformó la regulación de diversos ámbitos como la política. Los ciudadanos, no los súbditos, reclamaban derechos y deberes, y el Estado debía ofrecer unos instrumentos que reconociesen y que garantizasen sus libertades y derechos civiles y políticos. Fruto de lo anterior, como es sabido, el Estado Absoluto se transformó en el Estado de Derecho o Constitucional. Los súbditos pasaron a ser considerados como ciudadanos, y se consagró la división de poderes y el principio de legalidad.

Según el Diccionario de la Real Academia de la Lengua Española, en su edición 22a , la concertación es: "la acción de pactar, ajustar, tratar, acordar un negocio". También ésta es descrita, como un proceso de interlocución entre los actores sociales y el Estado, en un contexto donde los ciudadanos tengan derechos y deberes cumpliendo los requerimientos de las sociedades complejas. La concertación es pues un proceso complejo y de composición plural de actores.

Para Ángeles Barrios, "la concertación social sugiere la idea de un acuerdo conjunto, siendo la negociación de las partes en materias de un interés estratégico, sin exclusión del pacto político" (Barrios, 2014:7). Además esta autora, también señala que la concertación social, debe ser entendida como algo genérico y con un carácter inclusivo del diálogo o del pacto social, haciendo referencia además no solo a la voluntad de llegar a acuerdos entre partes, sino también y sobre todo, a la necesidad de que los acuerdos alcancen una legitimación política. Todo ello, con el convencimiento de los efectos positivos y pacificadores de la concertación para los actores involucrados y, en definitiva beneficiosos para reducir el conflicto social y facilitar a su vez la gobernabilidad del sistema, equilibrando sus necesidades entre sus actores implicados. La finalidad pues para esta autora de la concertación social, sería el logro de la "homeostasis" social y política.

No obstante, este concepto antedicho hay que relacionarlo también junto al proceso de actuación de los actores sindicales y patronales. La concertación social, es pues un término parecido o similar, pero no idéntico a otras acepciones tales como negociación colectiva, pacto social, acuerdo social, consenso social y diálogo social.

La situación de conflicto vivido durante la Segunda Guerra Mundial, inspiró el estudio de nuevas técnicas de negociación y acuerdo (ver: Habermas, 1987). El diálogo social parte de una relación entre actores no autoritaria, flexible y predispuesta a escuchar y ser escuchado, y compartir todas las cuestiones, dudas o reflexiones que se produzcan durante la negociación.

Algunos autores entienden por diálogo social, aquellas conversaciones formales e informales, realizadas por consulta, información o negociación entre los actores básicos siguientes: la Sociedad Civil y el Estado. Se entendería por Sociedad Civil, las empresas, los sindicatos, las asociaciones, las Organizaciones no gubernamentales,... con la finalidad de analizar conjuntamente, en colaboración del Estado o no, aquellos problemas sociales pendientes de lograr una solución. Estos grupos pueden aportar visiones diferentes y complementarias para abordar el tema y lograr un consenso, quedando obligados a su cumplimiento a través de un pacto social, fruto del previo acuerdo y diálogo.

El diálogo es ante todo y sobre todo, un aspecto y un proceso de los períodos democráticos, fruto de la participación social representativa. Esta es una forma de acción social. La participación social se analizará sesgadamente desde la pertenencia a una 
organización, tratándose de una participación activa o pasiva o incluso de una participación "fraccionada" o "del instante solidario", caracterizada por las siguientes notas:

a) en primer lugar, existe la participación activa que incluye a las personas que pertenecen a una misma organización, y que colaboran económicamente a través del pago de una cuota, además de entregar su tiempo gratuitamente a veces, para implicarse activamente en las actividades que la organización proponga, ya que se comparten unos mismos valores, y los ponen en práctica en común;

b) en segundo lugar, existe la participación pasiva o de solidaridad a distancia; que describe una forma de colaboración telemática y de sostenimiento, ya que únicamente los miembros participan con la aportación económica a la organización. Éstos creen en la causa, y en la lucha por determinados valores, que desarrolla la organización, y que el Estado no resuelve;

c) en tercer lugar, existe la llamada participación "fracturada", que consiste en colaborar en las actividades llevadas a cabo por la organización, y se rechaza la idea de ser etiquetado/a como afiliado/a o socio/a. El partícipe en este modo de participación, tiene la idea y la intención de mantenerse libre, sin pertenecer a ningún grupo, y sin sentirse cohibido/a por la decisión de la mayoría del grupo; y

d) por último, existe la denominada participación "del instante solidario".

Esta participación y colaboración, de carácter parcial y temporal, se caracteriza porque sus seguidores no están afiliados a ninguna organización, ni realizan ningún tipo de voluntariado o actividad, como miembros afiliados formales, sin abonar ninguna cuota. Estos individuos, de forma ocasional colaboran en las campañas, y además, a veces apoyan éstas de forma económica.

La participación es un elemento que ayuda también, a conocer el índice de solidaridad de la sociedad y es expresión del "capital social"; lo cual es resultado de los valores intrínsecos de los individuos, pero también de los compartidos socialmente, los cuales son asimilados durante todo el trayecto vital de la persona.

\section{EL DIÁLOGO CIVIL Y EL PARTENARIADO EN LA ACTUALIDAD}

En la actualidad, el "diálogo social" queda reducido al binomio Estado-empresario /trabajador, sin integrar otros actores de la sociedad civil. El "diálogo civil" viene a suplir deficiencias que no cubre el "diálogo social", sin embargo, es mucho más nuevo y no está consolidado ni tiene estructuras de funcionamiento como el anterior. También se habla del "diálogo institucional", que se da entre instituciones que representan a la sociedad, o entre el Estado y los agentes sociales más significativos. Hasta la fecha, cuando se habla de diálogo con el Poder Público se trata más de una consulta que de una "negociación sistemática" que incluya la planificación, la ejecución, el seguimiento y la evaluación de lo acordado.

El reconocimiento del Tercer Sector como interlocutor en el diálogo institucional, constituye a su vez un desafío en la actualidad pero de gran relevancia si lo que queremos es incluir a la sociedad civil organizada en el diálogo institucional y en la planificación de políticas públicas. El Tercer Sector, por sus características específicas, es un poderoso actor económico y social que supera el clásico esquema de la sociedad industrial. Un problema al que nos enfrentamos es que se pretenda incluir en el concepto de diálogo civil precisamente a las organizaciones empresariales, ya que la "sociedad civil" es "conjunto de todas las estructuras organizativas cuyos miembros prestan servicio al interés general por medio de un procedimiento democrático basado en el discurso y el consenso y sirven como mediadores 
entre las autoridades públicas y los ciudadanos", según lo define el Consejo Europeo Social y Económico.

Sin embargo, el problema más importante es que no existen mecanismos constituidos que orienten el diálogo y no se están construyendo, a pesar de que sólo la sociedad civil puede aportar una contribución a largo plazo. No obstante, se están generando plataformas que asocian a diversos actores según su especialización en su afán de coordinarse para obtener una voz común que aporte soluciones y propuestas a algunos problemas sociales, por ejemplo el Social Economy Europe (SEE), que es la integración de todos los actores del Tercer Sector a nivel europeo. Esta plataforma supone un gran paso integrador en planteamientos.

De todos modos, es necesario construir un partenariado real, entre los Poderes Públicos y el Tercer Sector, ya que ambos comparten objetivos comunes. El partenariado exige que cada actor sea mutuamente reconocido en la acción, planificación, ejecución y evaluación de las acciones propuestas. En la actualidad es difícil que se establezca porque no existen las condiciones necesarias pero la necesidad de establecer un partenariado auténtico sale a la palestra cada vez que se discute cómo mejorar la eficacia de lo público.

\section{LOS RESULTADOS Y LAS PRINCIPALES RECOMENDACIONES}

\subsection{Las principales recomendaciones para el cambio de paradigma}

Es necesario superar el estrecho diálogo social, que impide cerrar la brecha entre lo público y la sociedad. Para cumplir este objetivo, es necesario que los Poderes Públicos se comprometan a la creación de plataformas de diálogo con la sociedad que eviten que todo siga igual, así como estrategias para trabajar en partenariado.

El Tercer Sector, por su lado, debe comprometerse con la creación de estas plataformas y reubicarse en el contenido social global, yendo más allá de sus propias áreas de trabajo construyendo formas de comprender y actuar más acordes con las necesidades sociales en contextos concretos, además de superar sus propias inercias, desequilibrios personalistas y aprender a trabajar en cooperación.

\subsection{Las principales recomendaciones operativas para los Poderes Públicos}

Los objetivos económicos absorben en gran medida la fuerza de la gestión pública. Lo que es normal si estos objetivos económicos estuviesen enfocados a apoyar objetivos sociales. La raíz de la exclusión social se halla precisamente en que lo económico no engloba lo social, sino que lo contrapone en su objetivo de maximizar los beneficios sin atender cómo se realiza y las consecuencias que de ellos se derivan. Por ello, es preciso regular más y mejor, que los Poderes Públicos se aseguren de que se cumplen las normas y que la oferta laboral asuma su responsabilidad en la construcción de la cohesión social. Las recomendaciones que van en este sentido son:

1) Ubicar la economía dentro de la política, y no al revés, la política en el marco económico. La globalización económica ha relegado el poder político a los contextos locales, con lo que se ha supeditado a lo económico. Pero este traspaso de poder de lo político a lo financiero significa que las políticas que procuren regular la actividad económica, y la oferta laboral en concreto, se harán siempre desde objetivos meramente económicos (maximización del beneficio) sin tener en cuenta los objetivos sociales.

La finalidad de la economía, según se definía originariamente en el mundo académico, es: cubrir las necesidades humanas, contribuir al bienestar de la población, y era definida como 
la Ciencia que se dedicaba a la creación, distribución y consumo inteligente de la riqueza. Sin embargo, en la actualidad, medimos sólo el medio para cumplir estos objetivos: el PIB, en lugar de medir el fin: su utilidad social. Por lo que se supedita lo político a lo económico, dando como resultado que nunca se contemple la necesidad de generar cohesión social, o integrar laboralmente a las personas, además de que asistimos con naturalidad a una inversión en la cual son los "técnicos" -o controladores del sistema económico- los que dan respuestas a la sociedad en lugar de hacerlo los políticos.

2) Crear unos instrumentos de financiación que faciliten la potencialidad asociativa de la sociedad civil, con el objetivo de crear capital social y generar plataformas representativas con las que diseñar políticas públicas.

3) Sistematizar la publicación de datos (como calidad de vida, enseñanza, sanidad pública, protección social, empleo, pobreza, etc.) que permitan un seguimiento de la evolución de los desequilibrios sociales en su relación con el crecimiento económico. Esta es una medida necesaria para visibilizar lo que ocurre en el entorno (y no ocultarlo u olvidarse de ello) y poder hacer un seguimiento de la relación entre cohesión social y economía.

4) Es necesario que se fomente la formación y profesionalizar el trabajo de la sociedad civil, así como crear economías de escala que faciliten sinergias operativas e intercambio de buenas prácticas.

\subsection{Las principales recomendaciones para los actores del Tercer Sector}

1) Es importante profesionalizar la gestión del Tercer Sector compatibilizando el voluntariado con la gestión profesional. Aunque las organizaciones de la sociedad civil se especializan según su objeto social, la eficacia de su tarea requiere cada vez más de comportamientos profesionales en un sistema social que tiene más fuerza para generar exclusión que la acción que pretende neutralizarlo. Por ello, se ha de profundizar en la formación, en la mejor compatibilidad del trabajo voluntario y profesional, así como provocar acciones que generen sinergias, economías de escala e intercambien buenas prácticas.

2) Compatibilizar la especialización propia y la generación de plataformas integradoras que generen opinión común, y que ésta se pueda trasladar a la sociedad con la finalidad de crear criterios sobre el modelo social. Pero además, es especialmente importante para funcionar como "lobby" ante los Poderes Públicos e influir en la construcción de políticas públicas y la generación de normativas que afecten al modelo social por el cual trabajan.

3) Comunicar con la sociedad para romper la invisibilidad de estos actores. De manera que es necesario establecer políticas de comunicación con la sociedad que ayuden a trasladar planteamientos, inquietudes, criterios y formas de acción o propuestas. Todo ellos con el objetivo de fomentar una cultura socializadora que permita una sociedad más justa y solidaria.

4) Crear un observatorio que analice la cohesión social y la acción del mercado sobre el modelo social. Un observatorio que analice cuestiones como las acciones que provocan exclusión e inclusión, la pluralidad en el comportamiento económico y empresarial, o las fuerzas que rechazan esta pluralidad con planteamientos únicos, etc. pueden aportar propuestas de acciones correctoras importantes para la construcción de un modelo social, además de que puede ser un sistema fiable de datos estadísticos que visibilicen el Tercer Sector.

5) Las organizaciones del Tercer Sector -y la sociedad civil- deberían intervenir y desarrollar el concepto de Responsabilidad Social para que no se desvíe de su propósito ni quede en el olvido por parte de las organizaciones empresariales. 
6) Sería importante también, crear instrumentos de control de calidad y transparencia para impedir que se apropien de este tipo de marca (Tercer Sector), y la manipulen, aquellos que actúan con objetivos contrarios, incrementando de esta manera las barreras al intrusismo e incrementando la visibilidad y la credibilidad del sector.

\section{CONCLUSIONES}

Frente al sometimiento a "la racionalidad de los mercados", se aboga desde diversas corrientes por una supeditación del mercado a la racionalidad político democrática, es decir, a la voluntad social, como única manera de reequilibrar las relaciones de poder y hacer que el sistema funcione al servicio del interés general. El desarrollo democrático es un recurso para avanzar en mejoras de carácter instrumental que contribuyan a la sociedad, para lo que se reclama la participación ciudadana y la democracia participativa y directa. El partenariado Estado/Tercer Sector, podría significar un avance importante en este desarrollo y en la creación de una nueva gobernanza más inclusiva, más dinámica y más transparente y responsable.

\section{BIBLIOGRAFÍA}

ALÓS, R. y JÓDAR, P. (1996). "Nuevos cambios y viejas divisiones. Globalización del Capital y segmentación del trabajo". V Jornadas de Economía Crítica: La Crisis del Estado de Bienestar y la Unión Europea. Santiago de Compostela.

BARRIOS ALONSO, A. (2014). Por la razón y el derecho. Historia de la negociación colectiva en España (1850-2012). Granada: Comares.

CANALES ALIENDE, J. M. (2002). Lecciones de Administración y Gestión Pública. Alicante: Editorial Universidad de Alicante.

CASTRO SANZ, M. de (2010). "Las relaciones entre Tercer Sector y Poderes Públicos", Cuadernos de Debate 7, VII Foro del Tercer Sector. Fundación Luis Vives.

CIRIEC (2007). La Economía Social en la Unión Europea, Comité Económico y Social Europeo Unión Europea. Bruselas.

CIRIEC (2000). The Enterprises and Organizations of the Third System: A strategic challenge for employment. Liege: CIRIEC (Centre International de Recherches et d'Information sur l'Economie Publique, Sociale et Coopérative) - Directorate General V of the European Union.

DEFOURNY, J.; DEVELTERE, P. y FONTENAOU, B. (1999). Social Economy - North and South, HIVA. UK: Leuven.

GEORGE, S. (1999). "A short history of Neoliberalism. Twenty years of elite Economics and emerging opportunities for structural change", Conference on Economic Sovereignty in a Globalising World, Bangkok, 24-26 Marzo.

GÓMEZ CALVO, V. (2013). "Acercamiento a las prácticas de la Economía Solidaria, la Economía Social y la Economía del Bien Común, ¿qué nos ofrecen?”. Barataria. Revista Castellano-Manchega de Ciencias Sociales, 15: 111-124

HABERMAS, J. (1987). Teoría de la acción comunicativa. Madrid: Taurus.

MACEWAN, A. (2001). ¿Neoliberalismo o Democracia? Estrategias y alternativas económicas para el siglo XIX. Barcelona: Ediciones Octaedro e Intermón Oxfam.

MASSÉ, P. (1968). El plan o el antiazar. Barcelona: Nueva colección Labor.

NAVARRO, V. (2006). El subdesarrollo social de España. Causas y consecuencias. Madrid: Anagrama.

POLANYI, K. (1944). The Great Transformation. USA: Second Beacon Paper Edition.

REINERT, E.S. (2007). La globalización de la pobreza. Barcelona: Crítica. 


\section{Breve currículo:}

\section{Verónica Gómez Calvo}

Profesora Titular de la Universidad de Nevada (Reno, EEUU) en su programa de estudios en el extranjero USAC-Alicante sito en la Universidad de Alicante y miembro del Grupo Permanente de Investigación en el Observatorio Lucentino de Administración y Políticas Públicas Comparadas de la Universidad de Alicante. Es Doctora en Socio-economía, Especialista Universitario en Cooperación Internacional para el Desarrollo por la Universidad de Alicante y Licenciada en Filosofía pura por la Universidad de Deusto.

\section{Adela Romero Tarín}

Doctora en Derecho, Programa de Estudios Políticos y Constitucionales. Profesora del Área de Ciencia Política y de la Administración de la Universidad de Alicante. Máster en Gestión Pública y Dirección de Entidades Territoriales (2008), cursos de especialización en; Gestión administrativa de las ciudades y cursos de especialización en políticas culturales en la UOC (2010). Cursos de formación en la ECPR Summer School (Essex, 2011), ECPR Winter School (Viena, 2013) y EMAS (Salamanca, 2010). Ponente en congresos y seminarios nacionales e internacionales. Publicaciones en revistas, capítulos de libro y coordinación de revistas. Colaboración en Proyectos de investigación nacionales e internacionales. Especialista en temas de Gobierno Local, Políticas Públicas Urbanas, Gobernanza Urbana. 